\title{
A Rapidly-Incremented Tethered-Swimming Test for Defining Domain-Specific Training Zones
}

\author{
by \\ Dalton M. Pessôa Filho ${ }^{1,2}$, Leandro O.C. Siqueira ${ }^{1}$, Astor R. Simionato ${ }^{1}$, \\ Mário A.C. Espada ${ }^{3}$ Daniel S. Pestana ${ }^{4}$ Fred J. DiMenna ${ }^{5}$
}

The purpose of this study was to investigate whether a tethered-swimming incremental test comprising small increases in resistive force applied every 60 seconds could delineate the isocapnic region during rapidly-incremented exercise. Sixteen competitive swimmers (male, $n=11$; female, $n=5$ ) performed: (a) a test to determine highest force during 30 seconds of all-out tethered swimming $\left(F_{\text {avg }}\right)$ and the $\Delta F$, which represented the difference between $F_{\text {avg }}$ and the force required to maintain body alignment $(F$ base), and $(b)$ an incremental test beginning with 60 seconds of tethered swimming against a load that exceeded Fbase by $30 \%$ of $\Delta F$ followed by increments of $5 \%$ of $\Delta F$ every 60 seconds. This incremental test was continued until the limit of tolerance with pulmonary gas exchange (rates of oxygen uptake and carbon dioxide production) and ventilatory (rate of minute ventilation) data collected breath by breath. These data were subsequently analyzed to determine whether two breakpoints defining the isocapnic region (i.e., gas exchange threshold and respiratory compensation point) were present. We also determined the peak rate of $\mathrm{O}_{2}$ uptake and exercise economy during the incremental test. The gas exchange threshold and respiratory compensation point were observed for each test such that the associated metabolic rates, which bound the heavy-intensity domain during constant-work-rate exercise, could be determined. Significant correlations (Spearman's) were observed for exercise economy along with (a) peak rate of oxygen uptake $(\rho=.562 ; p<0.025)$, and (b) metabolic rate at gas exchange threshold $(\rho=-.759 ; p<0.005)$. A rapidly-incremented tethered-swimming test allows for determination of the metabolic rates that define zones for domain-specific constant-work-rate training.

Key words: isocapnic region; gas exchange threshold; respiratory compensation point; exercise economy; constantwork-rate exercise; heavy intensity.

\section{Introduction}

In both theory and practice, it is accepted that endurance training for athletes should comprise time spent in different exercise-intensity zones that are based on definable physiological responses (Midgley et al., 2007). For example, a typical week for an endurance athlete might include both easy training in the moderateintensity domain where a metabolic steady state is achieved rapidly and steady training in the heavy domain where a steady state is attainable, although delayed (Jones and DiMenna, 2011). Furthermore, training at intensities where a steady state is unattainable (e.g., tempo training in the severe domain and/or interval training with work intervals in the extreme domain) might also comprise part of the athlete's weekly load (Jones

\footnotetext{
1 - Institute of Biosciences, São Paulo State University (UNESP)/Rio Claro, Brazil.

2 - College of Sciences, São Paulo State University (UNESP)/Bauru, Brazil.

3 - Institute Polytechnical Septúbal, Portugal.

4 - Association of Aquatic Sports (ABDA) at Bauru, Brazil.

5 - Teachers College, Department of Biobehavioral Sciences, Columbia University, New York, USA.
} 
and DiMenna, 2011). It is, therefore, important to quantify the metabolic rates that separate athletes' exercise-intensity domains so that domain-specific training can be prescribed.

In 1981, Whipp et al. developed an incremental cycle-ergometer test where the work rate was applied as a smooth function of time (i.e., as a ramp; e.g., one watt every two seconds) (Whipp et al., 1981). Unlike slowly-incremented tests (e.g., traditional step tests with prolonged stages), this test allowed for clear delineation of the isocapnic region that separated two thresholds that could be identified from gas exchange and ventilatory data during incremental exercise (referred to in this article as the gas exchange threshold and respiratory compensation point; GET and RCP, respectively). Furthermore, small increments in the work rate that were continuously applied allowed for precise determination of the metabolic rates (i.e., rates of oxygen uptake) at each of these breakpoints (i.e., $\dot{\mathrm{V}} \mathrm{O}_{2 \mathrm{GET}}$ and $\dot{\mathrm{V}} \mathrm{O}_{2 \mathrm{RCP}}$ ). These increased aspects of sensitivity are important because $\dot{\mathrm{V}}_{2 \mathrm{GET}}$ and $\dot{\mathrm{V}} \mathrm{O}_{2 \mathrm{RCP}}$ are aligned with the metabolic rates that serve as lower and upper boundaries for the heavy-intensity domain during constant-workrate exercise (Jones and Poole, 2005; Keir et al., 2015; Stanula et al., 2014; Whipp and Wasserman, 1972). Consequently, a rapidly-incremented test with small work-rate increases is useful for prescribing domain-specific training and it is, therefore, not surprising that variations of this cycling protocol are used in the athletic, clinical and research setting. However, athletes must be tested in their specific mode of exercise and this is particularly the case for swimmers (Pinna et al., 2013). Hence, it is important to develop rapidlyincremented protocols that allow for precise determination of $\dot{\mathrm{V}} \mathrm{O}_{2 \mathrm{GET}}$ and $\dot{\mathrm{V}} \mathrm{O}_{2 \mathrm{RCP}}$ to prescribe endurance swim training.

Incremental tests that are used to assess cardiorespiratory capacity during free swimming typically involve intervals of set distance (e.g., 200 $\mathrm{m})$ performed at progressively-increasing velocities (Fernandes et al., 2003, 2011; Ribeiro et al., 2015). This means that unlike the smoothramp cycling test described above, these tests consist of lengthy stages comprising large unequal work-rate increments. It is, therefore, not surprising that researchers typically use these tests to identify only one of the aforementioned thresholds (e.g., often referred to as the anaerobic threshold) (Fernandes et al., 2011; Ribeiro et al., 2015). Indeed, due to its very nature, it is unlikely that this type of testing can identify the isocapnic region to derive the three-phase model that is best suited for exercise prescription (Binder et al., 2008; Skinner and McLellan, 1980). However, it is difficult to envision how rapidly-incremented small symmetrical changes in the work rate can be applied during free swimming because control of pace is imprecise. One alternative is to perform stationary swimming against a resistive load that can be increased with greater precision (i.e., tethered swimming). Research has confirmed that the maximal rate of oxygen uptake $\left(\dot{\mathrm{V}}_{2 m a x}\right)$ derived from an incremental tethered-swimming test is highly correlated with and not significantly different from that which is measured during free swimming (Bonen et al., 1980). However, in that study, no attempt was made to discern the threshold(s) that was/were encountered as the resistive load was increased and a discontinuous protocol was used with work bouts of 2-4 minutes separated by recovery intervals of $\geq 5$ minutes of rest (Bonen et al., 1980). Consequently, it is unlikely that this test would have been sensitive to threshold differentiation. Nevertheless, the ability of the tethered methodology to serve as a swim ergometer raises the intriguing possibility that a rapidly-incremented protocol similar to that which is used for stationary cycling could be developed using this approach.

In addition to its ability to discriminate the phases in which bicarbonate buffering is/is not sufficient to maintain homeostatic blood $\mathrm{pH}$ during incremental exercise, the smooth-ramp rapidly-incremented cycling test allows for determination of $\dot{\mathrm{V}}_{2 \max }$ if the test is continued until the limit of tolerance (Bogaard et al., 2008; Whipp et al., 1981; Whipp and Wasserman, 1972). The rapidly-incremented nature of this test is also important in this regard because longer incremental protocols (e.g., $\geq 12$ minutes) can result in underestimation of $\dot{\mathrm{V}}_{2 \max }$ (Astorino et al., 2004; Yoon et al., 2007). This has resonance for swimming because the incremental protocol that is typically employed (Fernandes et al., 2003, 2011; Ribeiro et al., 2015) comprises 7-8 steps and, therefore, overall test duration can exceed 12 minutes. Despite its non-steady-state nature, the smooth-ramp test also reveals a $\dot{\mathrm{V}}_{2} /$ work-rate 
slope that serves as a measure of exercise economy (Whipp et al., 1981). It is generally believed that improving $\dot{\mathrm{V}}{ }_{2 m a x}, \dot{\mathrm{V}}{ }_{2 \mathrm{GET}} / \dot{\mathrm{VO}}_{2 \mathrm{RCP}}$ and exercise economy might each require different training strategies (Jones and Carter, 2000); hence, the information provided by this singular test can be used to tailor athletic training to the characteristics of a given athlete and to monitor specific training-induced changes.

The purpose of the present study was to investigate whether a rapidly-incremented tethered-swimming protocol with small increases in resistive force could be used to delineate the isocapnic region that separates $\dot{\mathrm{VO}}_{2 \mathrm{GET}}$ and $\dot{\mathrm{V}} \mathrm{O}_{2 \mathrm{RCP}}$. For this reason, we had competitive swimmers of both sexes perform a novel incremental tetheredswimming test comprising work-rate increments equivalent to $5 \%$ of the difference between the maximal resistive force against which they could swim and the force required to maintain body alignment applied every 60 seconds. We hypothesized that two distinct breakpoints in gasexchange/ventilatory kinetics would be identifiable such that $\dot{\mathrm{V}} \mathrm{O}_{2 \mathrm{GET}}$ and $\dot{\mathrm{V}} \mathrm{O}_{2 \mathrm{RCP}}$ could be determined. We also measured $\dot{\mathrm{V}} \mathrm{O}_{2 \text { peak }}$ and the $\dot{\mathrm{V}} \mathrm{O}_{2} /$ load slope to explore relationships that might be present between exercise economy and the other variables of aerobic fitness.

\section{Material and Methods}

\section{Participants}

Eleven male (mean \pm SD: age, $18 \pm 4 \mathrm{yr}$; stature, $1.80 \pm 0.07 \mathrm{~m}$; body mass, $72 \pm 10 \mathrm{~kg}$ ) and five female (age, $17 \pm 4 \mathrm{yr}$; stature, $1.66 \pm 0.06 \mathrm{~m}$; body mass, $61 \pm 10 \mathrm{~kg}$ ) swimmers volunteered to participate in this study. The swimmers were competitive at the regional/national level and had each accumulated at least three years of competition training. The subjects were required to give their written informed consent prior to initiation of testing after the experimental procedures, associated risks and potential benefits of participation had been explained. For subjects under the age of 18 ( $n=13$; range, 14-17 years), signed consent from a parent or guardian was also obtained. This study was approved by the São Paulo State University ethics committee. The subjects were instructed to: 1.) avoid strenuous exercise in the 24 hours preceding each testing session; and 2.) arrive at the pool in a rested and fully-hydrated state at least three hours postprandial. Subjects were also asked to refrain from stimulant beverages and alcohol for 24 hours prior to each test.

\section{Procedures}

The competitive swimmers who agreed to participate reported to the pool for testing on two different occasions separated by 48 hours. During the first visit, subjects were familiarized with tethered swimming after which they performed an all-out tethered-swim test. Results from this test were used to calculate the resistive forces that would be applied during the incremental test, which was performed during the second visit. Both tests were undertaken at the same time of day for a given subject in a semi-Olympic swimming pool with water temperature of $\sim 28^{\circ} \mathrm{C}$.

The all-out tethered-swim test was performed with a 4905-N load cell attached to the swimmer's hip by an inelastic rope. For this test, subjects swam all-out for 30 seconds using a full front-crawl stroke with the averaged peaks of the wave frequency from the force-time signal defined as the trial's average force. Subjects performed this test twice separated by 20 minutes of rest and the higher value for average force $\left(\mathrm{F}_{\text {avg }}\right)$ was recorded for further analysis. The load cell was calibrated for $100 \mathrm{~Hz}$ signal acquisition prior to each test and the acquired signal was smoothed by the manufacturer's software package (N2000PRO, Cefise). We then determined the difference between $F_{\text {avg }}$ and the force that was required to maintain the swimmer's body alignment prior to initiation of the all-out swim (i.e., baseline force production; Fbase) to derive $\Delta \mathrm{F}$. The $F_{\text {base }}$ and $\Delta \mathrm{F}$ for each subject were used to calculate the starting resistive force and the increments in resistive force that would be applied during the incremental test.

During the second visit to the pool, subjects completed a maximal incremental tethered-swim test to determine whether the isocapnic region during rapidly-incremented exercise could be delineated during swimming. For this test, a custom-built weight-bearing pulley-rope system similar to a power rack, but modified for instantaneous weight-plate loading $(\geq 0.4-\mathrm{kg}$ increments) was used (Figure 1). Test administrators loaded the weight plates onto the system's carriage manually after receiving time cues from an associate. As was the case for the allout tethered-swim test, the rope of the load- 
application system was attached to the subject's hip and the subject swam using the front-crawl style. Importantly, attachment of the rope in this manner allowed for the leg kick to be unimpeded while providing a near-horizontal opposing force which resulted in minimal alteration of the standard swimming posture. Subjects were instructed to swim at a sufficient rate to avoid rearward/forward displacement of their body position as load increments were applied during each stage. Stage length was 60 seconds. The initial stage was performed against a load that exceeded Fbase by $30 \%$ of $\Delta \mathrm{F}$ and from that point, each stage comprised a load increment of $5 \%$ of $\Delta \mathrm{F}$. Two markers on the bottom of the pool provided visual reference points that allowed the swimmers to maintain a relatively-fixed position (e.g., $\pm 1 \mathrm{~m}$ from the desired position) and the test was terminated at the point at which this was no longer possible despite strong verbal encouragement from the testers. Breath-by-breath pulmonary gas-exchange data were collected using a portable metabolic unit designed for cardiopulmonary exercise testing (CPET K4b2; Cosmed, Rome, Italy). For this assessment, subjects breathed through a snorkel apparatus (new AquaTrainer ${ }^{\circledR}$ ) that had been validated for pulmonary $\dot{\mathrm{V}}_{2}$ measurement during swimming (Baldari et al., 2013). Before each test, the unit was calibrated according to the manufacturer's recommendations. After this procedure prior to attachment of the rope, subjects rested quietly on the pool border for 10 minutes with gas-exchange data collected in order to establish baseline parameters. Breath-by-breath $\mathrm{V}_{2}$ data collected during the baseline and exercise periods were averaged over consecutive nine-second periods after being smoothed by the collection unit's software. $\dot{V}_{2 \text { peak }}$ was defined as the highest threepoint rolling average of consecutive nine-second $\dot{\mathrm{V}}_{2}$ values recorded prior to the limit of tolerance. The final three-point rolling average for each completed $60 \mathrm{~s}$ stage was used to determine the $\dot{\mathrm{VO}}_{2} /$ load slope via linear regression. When $\mathrm{VO}_{2}$ failed to increase by an appreciable amount (determined by visual inspection) for $\geq 2$ stages immediately prior to the limit of tolerance, a $\mathrm{VO}_{2}$ plateau was considered to be present and datum from that stage was removed from the fit. Attempts to identify both GET and RCP were made by consensus from a panel of independent reviewers experienced at making these determinations from a cluster of measurements. For GET, these included: 1.) the first disproportionate increase in the rate of carbon dioxide production $\left(\mathrm{VCO}_{2}\right)$ from visual inspection of individual plots of $\mathrm{VCO}_{2} \mathrm{vs}$. $\mathrm{VO}_{2} ; 2$.) an increase in the expired rate of ventilation $\left(\dot{\mathrm{V}}_{\mathrm{E}}\right) / \mathrm{VO}_{2}$ with no increase in $\dot{\mathrm{V}}_{\mathrm{E}} / \mathrm{V} \mathrm{CO}_{2}$; and 3) an increase in endtidal $\mathrm{O}_{2}$ tension with no fall in end-tidal $\mathrm{CO}_{2}$ tension. For RCP, criteria included: 1.) the first disproportionate increase in $\dot{V}_{\mathrm{E}}$ in relation to $\dot{\mathrm{V}} \mathrm{CO}_{2}$; and 2.) a fall in end-tidal $\mathrm{CO}_{2}$ tension.

\section{Statistical Analysis}

The $\dot{\mathrm{V}} \mathrm{O}_{2 \mathrm{GET}}, \dot{\mathrm{V}} \mathrm{O}_{2 \mathrm{RCP}}, \dot{\mathrm{V}} \mathrm{O}_{2 \text { peak }}$ and $\dot{\mathrm{V}}_{2} / \mathrm{load}$ slope are expressed as group mean $\pm \mathrm{SD}$. Spearman's correlation coefficients were used to assess relationships between the $\mathrm{VO}_{2} /$ load slope and $\dot{\mathrm{V}} \mathrm{O}_{2 \mathrm{GET}}, \dot{\mathrm{V}} \mathrm{O}_{2 \mathrm{RCP}}$ and $\dot{\mathrm{V}} \mathrm{O}_{2 \text { peak. }}$. In all cases, statistical significance was accepted at $p<0.05$.

\section{Results}

The Favg from the all-out tethered-swim test in absolute and relative (to body mass) terms for the entire group was $185 \pm 41 \mathrm{~N}$ and $2.7 \pm 0.6 \mathrm{~N} \cdot \mathrm{kg}$ ${ }^{1} \mathrm{BM}$, respectively. The Favg was $208 \pm 43 \mathrm{~N}(2.9 \pm$ $\left.0.6 \mathrm{~N} \cdot \mathrm{kg}^{-1} \mathrm{BM}\right)$ for male subjects and $140 \pm 18 \mathrm{~N}$ $\left(2.3 \pm 0.3 \mathrm{~N} \cdot \mathrm{kg}^{-1} \mathrm{BM}\right)$ for female subjects. The limit of tolerance during the maximal incremental tethered-swim test occurred during stage $9.1 \pm 2.0$ (range, 6-14 stages; only one test $\geq 11$ stages), which equated to the load of $71 \pm 10 \%$ of Favg. In absolute terms, the load on the final stage of the incremental test was $9.3 \pm 1.5 \mathrm{~kg}$ (range, 7.1-13.1 $\mathrm{kg}$ ) for the entire group and $10.0 \pm 1.3 \mathrm{~kg}(8.1-13.1$ $\mathrm{kg})$ and $7.7 \pm 0.5 \mathrm{~kg}(7.1-8.3 \mathrm{~kg})$ for male and female subjects, respectively. In conjunction with the near-horizontal line of pull of the opposition that was applied, these relatively low loads in relation to the subject's body mass (10-16\%) allowed for the standard swimming posture to be maintained with the minimal alteration (e.g., swimmers' feet were not pulled downward to an excessive degree when they reached the final stage). Four of the 16 incremental tests resulted in a $\mathrm{VO}_{2}$ plateau prior to the limit of tolerance. $\grave{\mathrm{V}}_{2 \text { peak }}$ in absolute and relative (to body mass) terms for the entire group was $3.4 \pm 0.6 \mathrm{~L} \cdot \mathrm{min}^{-1}$ and $49.4 \pm 6.3 \mathrm{ml} \cdot \mathrm{kg}^{-1} \mathrm{BM} \cdot \mathrm{min}^{-1}$, respectively. $\grave{\mathrm{VO}_{2 p e a k}}$ was $3.7 \pm 0.4 \quad \mathrm{~L} \cdot \mathrm{min}^{-1}(51.7 \pm 5.2$ $\mathrm{ml} \cdot \mathrm{kg}^{1} \mathrm{BM} \cdot \mathrm{min}^{-1}$ ) for male subjects and $2.7 \pm 0.1$ $\mathrm{L} \cdot \mathrm{min}^{-1}\left(44.4 \pm 5.8 \mathrm{ml} \cdot \mathrm{kg}^{-1} \mathrm{BM} \cdot \mathrm{min}^{-1}\right)$ for female subjects. 


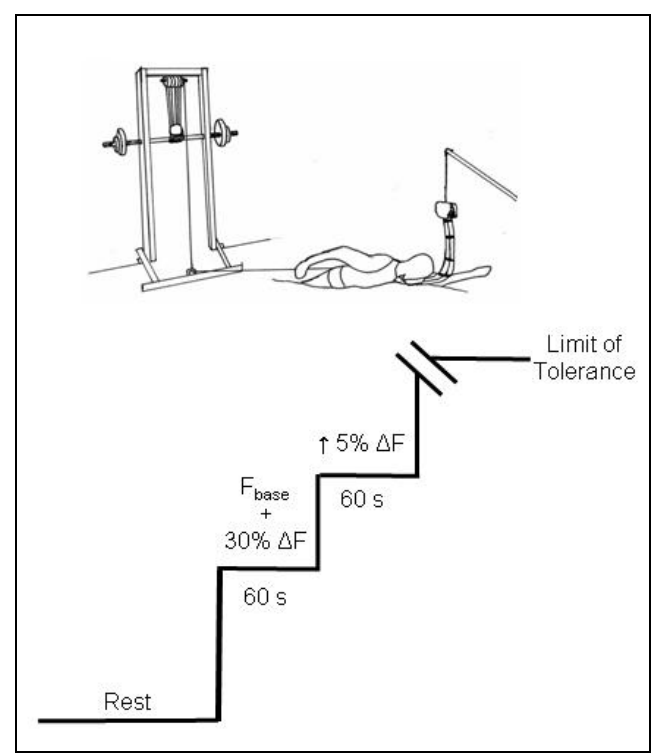

\section{Figure 1}

Depiction of the rapidly-incremented tethered-swimming protocol. Fbase is the opposing force that maintained the body position prior to loading and $\Delta F$ is the difference between Fbase and the highest force recorded for the subject.

\section{Figure 2}

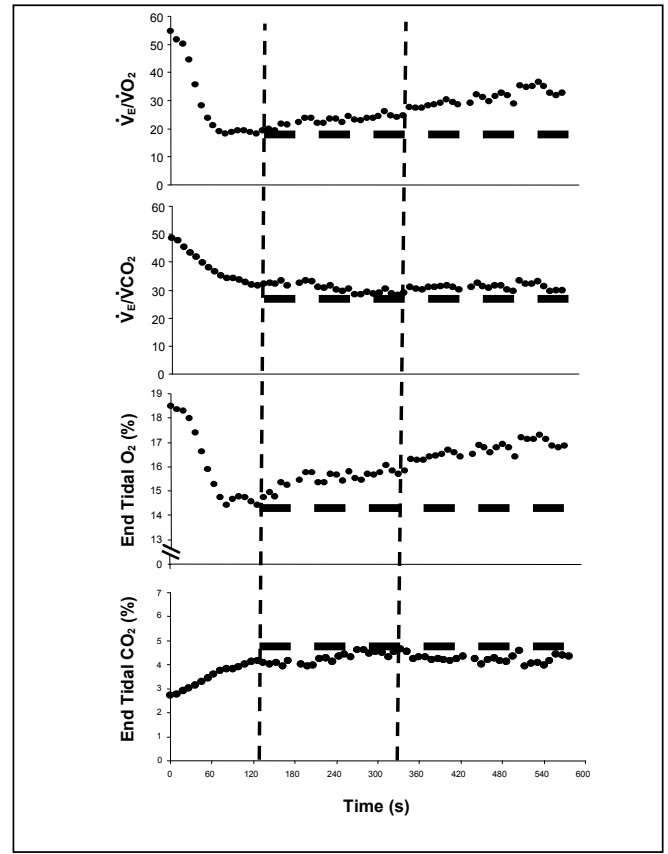

Gas exchange and ventilatory responses for a representative subject during the test.

From left to right, vertical dashed lines are aligned with the GET and RCP, respectively. Horizontal dashed lines are positioned at the nadir (top three panels) or apex (bottom panel) of data points. 


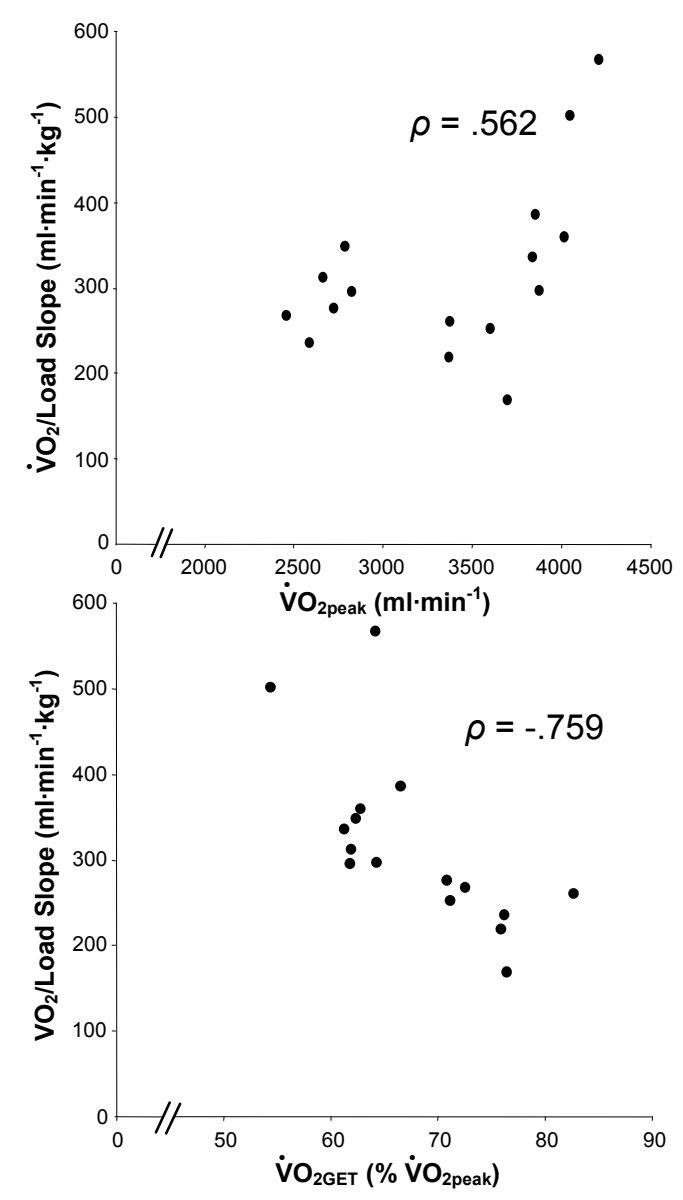

Figure 3

Significant correlations between $\mathrm{VO}_{2} /$ load slope and the peak rate of $\mathrm{O}_{2}$ uptake (Panel $A$ ) and the rate of $\mathrm{O}_{2}$ uptake at the GET relative to peak (Panel B).

All 16 of the maximal incremental swim tests were characterized by an isocapnic region such that $\dot{\mathrm{V}}_{2 \mathrm{GET}}$ and $\dot{\mathrm{V}} \mathrm{O}_{2 \mathrm{RCP}}$ could be identified (see Figure 2). Specifically, the $\dot{V}_{2 \text { GET }}$ in absolute and relative (to body mass) terms for the entire group was $2.3 \pm 0.4 \mathrm{~L} \cdot \mathrm{min}^{-1}$ and $33.6 \pm 5.8 \mathrm{ml} \cdot \mathrm{kg}^{-1} \mathrm{BM} \cdot \mathrm{min}^{-}$ 1 , respectively. The $\dot{\mathrm{V}} \mathrm{O}_{2 \mathrm{GET}}$ was $2.5 \pm 0.3 \mathrm{~L} \cdot \mathrm{min}^{-1}$ $\left(35.3 \pm 5.1 \mathrm{ml} \cdot \mathrm{kg}^{-1} \mathrm{BM} \cdot \mathrm{min}^{-1}\right)$ for male subjects and $1.8 \pm 0.1 \mathrm{~L} \cdot \mathrm{min}^{-1}\left(29.9 \pm 6.0 \mathrm{ml} \cdot \mathrm{kg}^{-1} \mathrm{BM} \cdot \mathrm{min}^{-1}\right)$ for female subjects. $\mathrm{VO}_{2 R C P}$ in absolute and relative (to body mass) terms for the entire group was $3.0 \pm$ $0.5 \mathrm{~L} \cdot \mathrm{min}^{-1}$ and $43.9 \pm 5.8 \mathrm{ml} \cdot \mathrm{kg}^{-1} \mathrm{BM} \cdot \mathrm{min}^{-1}$, respectively. $\mathrm{VO}_{2 \mathrm{RCP}}$ was $3.3 \pm 0.3 \mathrm{~L} \cdot \mathrm{min}^{-1}(45.8 \pm$ $\left.5.1 \mathrm{ml} \cdot \mathrm{kg}^{-1} \mathrm{BM} \cdot \mathrm{min}^{-1}\right)$ for male subjects and $2.4 \pm 0.2$
$\mathrm{L} \cdot \mathrm{min}^{-1}\left(39.6 \pm 5.6 \mathrm{ml} \cdot \mathrm{kg}^{-1} \mathrm{BM} \cdot \mathrm{min}^{-1}\right)$ for female subjects. $\dot{\mathrm{V}} \mathrm{O}_{2 \mathrm{GET}}$ and $\dot{\mathrm{V}} \mathrm{O}_{2 \mathrm{RCP}}$ occurred at $68 \pm 8 \%$ and $89 \pm 4 \%$ of $\dot{V}_{2 \text { peak, }}$ respectively.

The $\dot{\mathrm{VO}}_{2} /$ load slope in absolute and relative (to body mass) terms for the entire group was $317 \pm 102 \mathrm{ml} \cdot \mathrm{min}^{-1} \cdot \mathrm{kg}^{-1}$ and $4.7 \pm 1.2 \mathrm{ml} \cdot \mathrm{kg}^{-1-}$ $\mathrm{BM} \cdot \mathrm{min}^{-1} \cdot \mathrm{kg}^{-1}$, respectively. The $\mathrm{VO}_{2} /$ load slope was $329 \pm 120 \mathrm{ml} \cdot \mathrm{min}^{-1} \cdot \mathrm{kg}^{-1}(4.6 \pm 1.4 \mathrm{ml} \cdot \mathrm{kg}$ ${ }^{1} \mathrm{BM} \cdot \mathrm{min}^{-1} \cdot \mathrm{kg}^{-1}$ ) for male subjects and $291 \pm 43$ $\mathrm{ml} \cdot \mathrm{min}^{-1} \cdot \mathrm{kg}^{-1}\left(4.8 \pm 0.6 \mathrm{ml} \cdot \mathrm{kg}^{-1} \mathrm{BM} \cdot \mathrm{min}^{-1} \cdot \mathrm{kg}^{-1}\right)$ for female subjects. The correlation-coefficient values for the linear fits to the $\mathrm{VO}_{2} /$ load data ranged from 0.828 to 0.995 (mean $\pm \mathrm{SD}, 0.958 \pm 0.042$ ). The $\mathrm{VO}_{2} /$ load slope in absolute terms was positively 
correlated with the absolute $\mathrm{V}_{\text {2peak }}(\rho=.562 ; p<$ $0.025)$ and negatively correlated with the percentage of $\dot{\mathrm{V}}_{2 \text { peak }}$ at which $\dot{\mathrm{V}} \mathrm{O}_{2 \mathrm{GET}}$ occurred $(\rho$ $=-.759 ; p<0.005$ ) (Figure 3; top and bottom panel, respectively). There were no significant correlations between the $\mathrm{VO}_{2} /$ load slope and the metabolic rate at RCP. When analyzed according to sex, the negative correlation between the $\dot{\mathrm{VO}} 2 /$ load slope and the percent of $\dot{\mathrm{VO}}_{2 \text { peak }}$ at which V $\mathrm{O}_{2 \mathrm{GET}}$ occurred was present in both sexes whereas the positive correlation between $\mathrm{VO}_{2} /$ load slope and $\dot{\mathrm{V}} \mathrm{O}_{2 \text { peak }}$ remained significant only for male subjects.

\section{Discussion}

The main finding from this investigation is that a tethered swimming incremental protocol comprising relatively small work-rate increments (e.g., a resistive load increase of as little as $0.4 \mathrm{~kg}$ ) applied every 60 seconds was sufficiently rapid to allow delineation of the isocapnic region that can be present during incremental exercise. This means that the two distinct breakpoints in the gas exchange/ventilatory response that can be identified during incremental exercise (referred to in this article as gas exchange threshold and respiratory compensation point, GET and RCP, respectively) were able to be determined from this singular test. We also calculated the $\dot{\mathrm{V}}_{2} / \mathrm{load}$ slope as a measure of exercise economy and found that for the competitive swimmers we assessed, this slope was positively correlated with $\dot{\mathrm{V}}_{2 \text { peak, }}$ but negatively correlated with the metabolic rate at GET expressed relative to $\dot{V}_{2}$ peak. This implies that the magnitude of $\dot{V}_{2 \mathrm{GET}}$ relative to maximal capacity can exert a considerable effect on endurance-swimming performance irrespective of $\dot{\mathrm{V}} \mathrm{O}_{2 \max }$ per se.

In the present study, we tested a novel incremental protocol that allowed for determination of $\dot{\mathrm{V}} \mathrm{O}_{2 \text { peak, }} \dot{\mathrm{V}} \mathrm{O}_{2 \mathrm{GET}}, \dot{\mathrm{V}} \mathrm{O}_{2 \mathrm{RCP}}$ and exercise economy during tethered swimming. The test was novel because the increments were applied more rapidly than is typically the case during free swimming incremental protocols. For example, the protocol that is often used involves multiple even-paced 200-m swimming bouts ( $\mathrm{n} \mathrm{x}$ $200 \mathrm{~m}$ ) performed at increasing velocities beginning with an initial step at an easy pace and culminating with a step at maximal effort (Pyne et al., 2001). This type of testing is a modified version of the traditional step-incremental tests that are employed for cycling and running where stage length is fixed (e.g., 3-5 minutes) and the work rate is increased progressively. In addition to allowing for the determination of $\dot{\mathrm{V}}_{2 \max }$ at or near the limit of tolerance, these slowlyincremented tests were designed to provide information on metabolic responses to submaximal work; for example, the metabolic rate at which a sustained increase in blood lactate concentration above resting levels $\left(\geq \sim 1 \mathrm{mmol} \cdot \mathrm{L}^{-1}\right)$ is initially observed (the lactate threshold; LT) and the change in external work produced in relation to the change in energy expenditure for completion of a step (delta efficiency). Consequently, extended stage lengths were necessary so that a metabolic steady state could be achieved upon completion of each stage. However, it is now well established that rapid attainment of a steady state (e.g., in $\leq 2$ minutes) is only possible for work that is performed below LT with a steady state delayed for up to 15 minutes in what has been termed the heavy-intensity domain or even unattainable when higher work rates are encountered (Jones and Poole, 2005; Poole et al., 1988). Consequently, there is little rationale for prolonging each stage and, indeed, there are drawbacks with such an approach. For example, lengthy stage duration results in a prolonged overall test (e.g., $\geq 12$ minutes) that might prevent the attainment of $\dot{\mathrm{V}} \mathrm{O}_{2 \max }$ upon exhaustion (Astorino et al., 2004; Yoon et al., 2007). Furthermore, during a slowly-incremented test (e.g., stage duration $\geq 3$ minutes), non-invasive identification of LT via gas exchange/ventilatory changes corresponding to the non-metabolic production of $\mathrm{CO}_{2}$ is complicated by the fact that $\dot{V}_{\mathrm{E}}$ will increase disproportionately compared to $\dot{\mathrm{V}} \mathrm{CO}_{2}$ for all work rates above the LT (Whipp et al., 1989). Consequently, slowly-incremented tests will not allow for identification of the isocapnic region within which arterial partial pressure of $\mathrm{CO}_{2}$ is maintained above LT during incremental exercise (i.e., supra-LT work where compensatory hyperventilation is not yet required).

The range of metabolic rates within the isocapnic region during incremental exercise approximate those that comprise the heavyintensity domain during exercise performed at a constant rate of work (Jones and Poole, 2005; Keir et al., 2015; Whipp and Wasserman, 1972). This 
domain is bounded on its upper end by the maximal lactate steady state, which means that heavy-intensity exercise can be sustained for extended periods despite an elevation of bloodlactate concentration above resting levels (Espada et al., 2015; Jones and Poole, 2005). Importantly, it is believed that training within this domain enhances exercise economy and shifts both the LT and lactate turnpoint (i.e., the acceleration in blood lactate accumulation during incremental exercise that typically occurs around 2.5-4.0 $\mathrm{mmol} \cdot \mathrm{L}^{-1}$ ) to higher work rates (Jones and DiMenna, 2011). Defining these metabolic rates (e.g., in the present study, a range that spanned from $\sim 33.6$ to $\sim 49.4 \mathrm{ml} \cdot \mathrm{kg}^{-1} \mathrm{BM} \cdot \mathrm{min}^{-1}$ ) is, therefore, important because continuous training within the heavy domain would likely make up a good portion of an endurance swimmer's weekly training load.

Unlike the rapidly-incremented test that we employed, the $n \times 200 \mathrm{~m}$ slowly-incremented test provides rest periods between stages that allow for blood to be drawn. This means that estimation of GET and RCP via gas exchange/ventilatory data is not necessary because blood lactate dynamics can be assessed directly (Fernandes et al., 2011). However, this test is typically used to identify a singular anaerobic threshold, a term that is problematic because it has been used to describe both of the thresholds that are defined according to the three-phase model (Binder at al., 2008). Furthermore, Fernandes et al. (2011) used the conventional protocol to identify an individual anaerobic threshold that occurred at a blood lactate concentration of $\sim 2 \mathrm{mmol} \cdot \mathrm{L}^{-1}$. Given this level of blood lactate accumulation, this threshold was likely greater than the LT; hence, the associated metabolic rate would be greater than the one that defines the lower boundary of the heavy-intensity domain (i.e., $\dot{\mathrm{V}}_{2 \mathrm{GET}}$ ). Moreover, they reported that blood lactate concentration at the individual anaerobic threshold was significantly less than that which was present during constant-work-rate swimming at the maximal lactate steady state $(\sim 3$ $\left.\mathrm{mmol} \cdot \mathrm{L}^{-1}\right)$. This means that the associated metabolic rate was less than the critical metabolic rate that serves as the heavy domain's upper boundary ( $\dot{\mathrm{V}}_{2 \mathrm{RCP}}$ ) (Keir et al., 2015). Ribiero et al. (2015) also reported a singular anaerobic threshold for subjects performing the $\mathrm{n} \times 200 \mathrm{~m}$ protocol regardless of whether a direct (blood lactate concentration) or indirect (gasexchange/ventilation) measurement was used for the determination.

In addition to $\dot{\mathrm{V}} \mathrm{O}_{2 \mathrm{GET}}, \dot{\mathrm{V}} \mathrm{O}_{2 \mathrm{RCP}}$ and $\dot{\mathrm{V}} \mathrm{O}_{2 \text { peak, }}$ we also calculated the $\dot{\mathrm{V}}_{2} /$ load slope during the rapidly-incremented test as a proxy measure of swimming efficiency. This slope, which represents the inverse of delta efficiency, provides an estimate of exercise economy that can be derived from rapidly-incremented tests that take place entirely in the non-steady state (Whipp et al., 1981). Our results showed considerable variance in this parameter with a range from 2.3 to 7.0 $\mathrm{ml} \cdot \mathrm{kg}^{-1} \mathrm{BM} \cdot \mathrm{min}^{-1}$ per kilogram of the load applied in the 16 swimmers that we tested. However, a consistent feature was that swimmers with a greater oxidative capacity possessed lower exercise economy. Indeed, we observed a positive correlation between $\dot{\mathrm{V}} \mathrm{O}_{2 \text { peak }}$ and the $\dot{\mathrm{VO}}_{2} / \mathrm{load}$ slope (Figure 3; top panel) which, at first glance, appears counterintuitive. However, an inverse relationship between $\dot{\mathrm{V}} \mathrm{O}_{2 \max }$ and exercise economy had previously been reported for professional cyclists during cycling (Lucia et al., 2002) and premenopausal women with a wide range of $\dot{\mathrm{V}}_{2 \max }$ values during graded treadmill walking (Hunter et al., 2005). Furthermore, in the latter study, the researchers used 31P magnetic resonance spectroscopy to assess oxidative capacity and exercise economy on the muscletissue level during isometric plantarflexion and much like the whole-body measurement, a similar inverse relationship was found (Hunter et al., 2005). Consequently, our findings for tethered swimming are in line with what has been shown for these other modes of exercise.

In the present study, we also found a significant relationship between $\dot{\mathrm{V}}_{2 \mathrm{GET}}$ stated as a percentage of $\dot{\mathrm{V}}_{2 \text { peak }}$ and the $\dot{\mathrm{V}}_{2} /$ load slope; however, this correlation was negative (Figure 3; bottom panel). Interestingly, it has been speculated that the inverse relationship between oxidative capacity and exercise economy might be attributable to a high proportion of type IIa fibers, which have lower exercise economy yet contribute significantly to $\dot{\mathrm{V}}_{2 \max }$ (Hunter et al.,

2005). Conversely, type I fibers, which also have a profound impact on $\dot{\mathrm{V}}_{2 \max }$, appear to possess high exercise economy (Coyle et al., 1992; Crow and Kushmerick, 1982) and it is these fibers that 
would likely contribute predominantly to all metabolic rates below $\dot{V}_{2 \text { GET }}$ (Henneman and Mendell, 1981). Consequently, our findings suggest that swimmers who can delay recruitment of higher-order fibers for a greater proportion of their overall capacity for work will be more economical during endurance swimming regardless of the magnitude of the $\dot{\mathrm{V}} \mathrm{O}_{2 \max }$ they possess. These results are consistent with the belief that training to improve exercise economy might require a different stimulus compared to training to improve $\dot{\mathrm{V}} \mathrm{O}_{2 \max }$; for example, domainspecific training at a heavy intensity for prolonged duration (i.e., steady training) to increase $\dot{V} \mathrm{O}_{2 \mathrm{GET}}$. This type of training could, therefore, have important practical implications being that exercise economy can account for large variations in performance for endurance athletes with similar $\dot{\mathrm{V}}_{2 \max }$ values (Conley and Krahenbuhl, 1980).

Collectively, the rapid incrementation and precise control of the load afforded by the tethered-swimming test we investigated make it suitable for discerning metabolic rates that bound the heavy-intensity domain. Consequently, unlike a free-swimming incremental protocol involving distance-controlled stages, this test provides information that is useful for domain-specific exercise prescription according to the three-phase model (Binder et al., 2008; Skinner and McLellan, 1980; Whipp et al., 1989). However, it is important to note that the degree to which the swimming technique employed during tethered swimming relates to that which is present during free swimming has been questioned (DominguezCastells and Arellano, 2012). Thus, it is possible that the parameters derived from this test might only apply to domain-specific training utilizing the tethered methodology. However, there is evidence to suggest similarities between measurements derived during tethered and free swimming. For example, Bonen et al. (1980) had swimmers perform an incremental tethered protocol (step increases in the load applied during 2-4 minute work bouts interspersed with $\geq 5$ minutes of rest) and found that the peak $\mathrm{VO}_{2}$ they observed was highly correlated with $(r>0.99)$ and not significantly different from the peak $\dot{\mathrm{VO}}_{2}$ recorded when subjects performed three bouts of $200 \mathrm{~m}$ free swims at increasing velocities (moderate, faster and all out). Interestingly, in a separate but related experiment, these authors also found a similar peak $\dot{\mathrm{V}}_{2}$ response during incremental tethered and flume swimming; however, both of these values were greater than the value observed during arm cranking (Bonen et al., 1980). This suggests that the tethered methodology achieved swim specificity that was not present with upper-body exercise per se. Moreover, Perandini et al. (2006) had subjects perform 3-4 constant-load tethered-swimming bouts to exhaustion and modelled the force/time data to reveal a critical force (i.e., force/time asymptote or y-intercept using hyperbolic and linear fits, respectively) that was significantly correlated with the critical velocity they estimated for free swimming $(\mathrm{r} \approx 0.90)$. However, $\dot{\mathrm{V}}_{2}$ was not measured in that study; hence, the degree to which the metabolic rates at the tetheredswimming critical force and free-swimming critical velocity were similar (e.g., presumably, a critical metabolic rate that is equivalent to $\dot{\mathrm{VO}}_{2 \mathrm{RCP}}$; Keir et al., 2015) could not be determined. Parameters derived from both incremental (Papoti et al., 2009) and constant-load all-out (Kalva-Filho et al., 2015; Papoti et al., 2010) tethered-swimming tests have also been shown to predict performance during free swimming. Finally, Matsumoto et al. (1999) used a discontinuous incremental-loading tethered protocol with fourminute stages to determine the LT in children with asthma (Matsumoto et al., 1999). Following this assessment, these researchers prescribed freeswim training according to the heart rate at LT and reassessed their subjects using the tethered methodology after six weeks. Importantly, the load at LT was increased by free-swim training, which suggests that a productive free-swim training regimen can be both prescribed and assessed via measurements made using the tethered methodology.

There are a number of limitations to this study that deserve mention. In addition to the inability to confirm that the parameters derived from this test are similar to those that would be identified during free swimming, we also did not verify that $\dot{\mathrm{V}} \mathrm{O}_{2 \mathrm{GET}}$ and $\dot{\mathrm{V}} \mathrm{O}_{2 R C P}$ approximate the metabolic rates at the lower and upper boundaries of the heavy-intensity domain during constant-workrate tethered swimming. While prior research suggests that this is the case for other forms of exercise (e.g., cycling and running; 
Jones and Poole, 2005; Keir et al., 2015; Whipp and Wasserman, 1972), future research should provide confirmation by having subjects perform the tethered incremental protocol in conjunction with a series of constant-work-rate bouts against a variety of loads to identify moderate/heavy and heavy/severe interfaces.

In conclusion, we demonstrated that an incremental tethered-swimming test with relatively small increases in the resistive load applied every 60 seconds was sufficiently sensitive to reveal the two gas exchange/ventilatory breakpoints (GET and RCP) defining the isocapnic region during incremental exercise. The metabolic rates at these thresholds serve as lower ( $\dot{\mathrm{V}}_{2 \mathrm{GET}}$ ) and upper ( $\left.\dot{\mathrm{V}}_{2 \mathrm{RCP}}\right)$ boundaries of the heavy-intensity domain during constant-work-rate exercise; hence, this singular test can be used to define three distinct exerciseintensity zones for domain-specific training. We also found a high negative correlation between exercise economy and GET suggesting that regardless of $\dot{\mathrm{V}}_{2 \max }$, increasing GET relative to maximal capacity can have important implications for endurance-swim performance. Training in the heavy-intensity domain provides a potent stimulus to achieve this objective. Future research should explore the degree to which these conclusions drawn from a tethered-swimming protocol are related to responses observed during free-swim training and competition.

\section{Acknowledgements}

The authors would like to thank the swimmers from the BTC team who participated in this study and Santander Totta (PROPG-UNESP) for the fellowship support. This work was supported by the Brazilian National Council for Scientific and Technological Development (CNPq; 479262/2013-6).

\section{References}

Astorino TA, Rietschel JC, Tam PA, Taylor K, Johnson, SM, Freedman TP, Sakarya CE. Reinvestigation of optimal duration of $\mathrm{VO}_{2 \max }$ testing. J Exerc Physiol Online, 2004; 7: 1-8

Baldari C, Fernandes RJ, Meucci M, Ribeiro J, Vilas-Boas JP, Guidetti L. Is the new AquaTrainer ${ }^{\circledR}$ snorkel valid for $\mathrm{VO}_{2}$ assessment in swimming? Int J Sports Med, 2013; 34: 336-344

Binder RK, Wonisch M, Corra U, Cohen-Solal A, Vanhees L, Saner H, Schmid JP. Methodological approach to the first and second lactate threshold in incremental cardiopulmonary exercise testing. Eur J Cardiovasc Prev Rehabil, 2008; 15: 726-734

Bogaard HJ, Woltjer HH, van Keimpema AR, Serra RA, Postmus PE, de Vries, PMJM. Comparison of the respiratory and hemodynamic responses of healthy subjects to exercise in three different protocols. Occup Med, 1996; 46: 293-98

Bonen A, Wilson BA, Yarkony M, Belcastro AN. Maximal oxygen uptake during free, tethered, and flume swimming. J Appl Physiol Respir Environ Exerc Physiol, 1980; 48: 232-235

Conley DL, Krahenbuhl GS. Running economy and distance running performance of highly trained athletes. Med Sci Sports Exerc, 1980; 12: 357-360

Coyle EF, Sidossis LS, Horowitz JF, Beltz JD. Cycling efficiency is related to the percentage of type I muscle fibers. Med Sci Sports Exerc, 1992; 24: 782-788

Crow MT, Kushmerick MJ. Chemical energetics of slow- and fast-twitch muscles of the mouse. J Gen Physiol, 1982; 79: 147-66

Dominguez-Castells R, Arellano R. Effect of different loads on stroke and coordination parameters during freestyle semi-tethered swimming. J Hum Kinet, 2012; 32: 33-41

Espada MC, Reis JF, Almeida TF, Bruno PM, Vleck VE, Alves FB. Ventilatory and Physiological Responses in Swimmers Below and Above Their Maximal Lactate Steady State. J Strength Cond Res, 2015; 29: 28362843

Fernandes RJ, Cardoso CS, Soares SM, Ascensão A, Colaço PJ, Vilas-Boas JP. Time limit and $\mathrm{VO}_{2}$ slow component at intensities corresponding to $\mathrm{VO}_{2 \max }$ in swimmers. Int J Sports Med, 2003; 24: 576-581 
Fernandes RJ, Sousa M, Machado L, Vilas-Boas JP. Step length and individual anaerobic threshold assessment in swimming. Int J Sports Med, 2011; 32: 940-946

Henneman E, Mendell LM. Functional organisation of motoneuron pool and its inputs. In: Handbook of Physiology I vol. II. VB Brooks, ed. Maryland, American Physiological Society, 423-507; 1981

Hunter GR, Bamman MM, Larson-Meyer DE, Joanisse DR, McCarthy JP, Blaudeau TE, Newcomer BR. Inverse relationship between exercise economy and oxidative capacity in muscle. Eur J Appl Physiol, 2005; 94: 558-568

Jones AM, Carter H. The effect of endurance training on parameters of aerobic fitness. Sports Med, 2000; 29: 373-86

Jones AM, DiMenna FJ. Cardiovascular Assessment and Aerobic Training Prescription. In: Strength and Conditioning: Biological Principles and Practical Applications. M. Cardinale, R. Newton and K. Nosaka, eds. London: John Wiley \& Sons, Ltd., 291-304; 2011

Jones AM, Poole DC. Introduction to Oxygen Uptake Kinetics. In: Oxygen Uptake Kinetics in Sport, Exercise and Medicine. AM Jones and DC Poole, eds. London: Routledge, 3-35; 2005

Kalva-Filho CA, Zagatto AM, Araújo MI, Santiago PR, da Silva AS, Gobatto CA, Papoti M. Relationship between aerobic and anaerobic parameters from 3-minute all-out tethered swimming and 400-m maximal front crawl effort. J Strength Cond Res, 2015; 29: 238-245

Keir DA, Fontana FY, Robertson TC, Murias JM, Paterson DH, Kowalchuk JM, Pogliaghi S. Exercise Intensity Thresholds: Identifying the Boundaries of Sustainable Performance. Med Sci Sports Exerc, 2015; 47:1932-40

Lucía A, Hoyos J, Pérez M, Santalla A, Chicharro JL. Inverse relationship between $\mathrm{VO}_{2 \max }$ and economy/efficiency in world-class cyclists. Med Sci Sports Exerc, 2002; 34: 2079-2084

Matsumoto I, Araki H, Tsuda K, Odajima H, Nishima S, Higaki Y, Tanaka H, Tanaka M, Shindo M. Effects of swimming training on aerobic capacity and exercise induced bronchoconstriction in children with bronchial asthma. Thorax, 1999; 54: 196-201

Midgley AW, McNaughton LR, Jones AM. Training to enhance the physiological determinants of longdistance running performance: can valid recommendations be given to runners and coaches based on current scientific knowledge? Sports Med, 2007; 37: 857-880

Papoti M, Vitório R, Araújo GG, Martins LEB, Cunha SA, Gobatto CA. Critical force during tethered swimming for the evaluation of aerobic capacity and prediction of performances in freestyle swimming. Rev. Bras. Cineantropom, 2010; 12: 14-20

Papoti M, Vitório R, Araújo GG, DaSilva ASR, Santhiago V, Martins LEB, Cunha SA, Gobatto CA. Determination of force corresponding to maximal lactate steady state in tethered swimming. Int $J$ Exerc Sci, 2009; 2: 269-279

Perandini LAB, Okuno NM, Kokubun E, Nakamura FY. Correlation between critical force and critical velocity and their respective stroke rates. Rev. Bras. Cineantropom, 2006; 8: 58-65

Pinna M, Milia R, Roberto S, Marongiu S, Olla S, Loi A, Ortu M, Migliaccio GM, Tocco F, Concu A, Crisafulli A. Assessment of the specificity of cardiopulmonary response during tethered swimming using a new snorkel device. J Physiol Sci, 2013; 63: 7-16

Poole DC, Ward SA, Gardner GW, Whipp BJ. Metabolic and respiratory profile of the upper limit for prolonged exercise in man. Ergonomics, 1988; 31: 1265-79

Pyne DB, Lee H, Swanwick KM. Monitoring the lactate threshold in world-ranked swimmers. Med Sci Sports Exerc, 2001; 33: 291-297

Ribeiro J, Figueiredo P, Sousa M, De Jesus K, Keskinen K, Vilas-Boas JP Fernandes RJ. Metabolic and ventilatory thresholds assessment in front crawl swimming. Sports Med Phys Fitness, 2015; 55: 701-707

Skinner JS, McLellan TM. The transition from aerobic to anaerobic metabolism. Res Q Exerc Sport, 1980; 51: 234-248 
Stanula A, Roczniok R, Maszczyk A, Pietraszewski P, Zając A. The role of aerobic capacity in highintensity intermittent efforts in ice-hockey. Biol. Sport, 2014; 31:193-199

Whipp BJ, Davis JA, Torres F, Wasserman, K. A test to determine parameters of aerobic function during exercise. J Appl Physiol Respir Environ Exerc Physiol, 1981; 50: 217-21

Whipp BJ, Davis JA, Wasserman K. Ventilatory control of the 'isocapnic buffering' region in rapidlyincremental exercise. Respir Physiol, 1989; 76: 357-367

Whipp BJ, Wasserman K. Oxygen uptake kinetics for various intensities of constant-load work. J Appl Physiol, 1972; 33: 351-356

Yoon BK, Kravitz L, Robergs R. $\mathrm{VO}_{2 \max }$, protocol duration, and the $\mathrm{VO}_{2}$ plateau. Med Sci Sports Exerc, 2007; 39:1186-1192

Zhang YY, Johnson MC, Chow N, Wasserman K. Effect of exercise testing protocol on parameters of aerobic function. Med Sci Sports Exerc, 1991; 23: 625-630

\section{Corresponding author:}

Fred DiMenna, Ph.D.

Department of Biobehavioral Sciences

Columbia University Teachers College

525 W. 120th Street

New York, N.Y. 10027

Phone: 212-678-3325/Fax: 212-678-3322

E-mail: DiMenna@exchange.tc.columbia.edu 